\title{
Prediction of a Movie's Success From Plot Summaries Using Deep Learning Models
}

\author{
You-Jin Kim \\ Jung-Hoon Lee \\ Department of Applied Data Science College of Computing \\ Sungkyunkwan University Sungkyunkwan University \\ Suwon-si, South Korea Suwon-si, South Korea \\ k01077679687@gmail.com vhrehfdl@gmail.com
}

\author{
Yun-Gyung Cheong \\ College of Computing \\ Sungkyunkwan University \\ Suwon-si, South Korea \\ a imecca@skku.edu
}

\begin{abstract}
As the size of investment for movie production grows bigger, the need for predicting a movie's success in early stages has increased. To address this need, various approaches have been proposed, mostly relying on movie reviews, trailer movie clips, and SNS postings. However, all of these are available only after a movie is produced and released. To enable a more earlier prediction of a movie's performance, we propose a deep-learning based approach to predict the success of a movie using only its plot summary text. This paper reports the results evaluating the efficacy of the proposed method and concludes with discussions and future work.
\end{abstract}

\section{Introduction}

Movie industry is a huge sector within the entertainment industry. The global movie box office revenue is predicted to reach nearly 50 billion U.S dollars in 2020 (Sachdev et al., 2018). With huge capital investments, the movie business is a high-risk venture (De Vany and Walls, 1999). Therefore, an early prediction of a movie's success can make a great contribution to the film industry, when post-production factors are unknown before the film's release. This task is extremely challenging, as the success of the movie should be determined based on the scenario or plot of the movie without using the post-production drivers such as actor, actress, director, MPAA rating and etc.

To address this issue, our work attempts to predict a movie's success from its textual summary. We used the CMU Movie Summary Corpus ${ }^{1}$, which contains crowd-sourced summaries from the real users. The success of a movie is assessed with the review scores of Rotten Tomatoes ${ }^{2}$, an American review-aggregation website

\footnotetext{
${ }^{1}$ http://www.cs.cmu.edu/ ark/personas/

${ }^{2}$ https://www.rottentomatoes.com/
}

for film and television. The scoring system utilizes two scores: the tomato-meter and the audience score. The tomato-meter score is estimated by hundreds of film and television critics, appraising the artistic quality of a movie. The audience score is computed by the collective scores from regular movie viewers.

In this paper we present a deep-learning based approach to classify a movie popularity and quality labels using the movie textual summary data. The primary hypothesis that we attempted to answer is to predict a movie's success in terms of popularity and artistic quality by analyzing only the textual plot summary.

The contributions of our research are as follows:

- To prepare a data set to define a movie's success

- To incorporate sentiment score in predicting a movie's success

- To evaluate the efficacy of ELMO embedding in predicting a movie's success

- To evaluate merged deep learning models (CNN and residual LSTM) in predicting a movie's success

\section{Our Approach}

Figure 1 illustrates the system architecture that classifies an input text as successful or nonsuccessful based on the critics score and the audience score.

The pre-processing step tokenizes the summary text into sentences. Then, the list of sentences are given to the ELMO embedding and the sentiment score extraction modules. The ELMO embedding module converts the sentences into word vectors. The sentiment score extractor generates a sentiment score that combines the positive and negative sentiment score of each sentence. Lastly, the two 


\begin{tabular}{|c|c|c|c|c|c|}
\hline & \multicolumn{2}{|c|}{ Train } & \multicolumn{2}{|l|}{ Test } & \multirow[b]{2}{*}{ Total } \\
\hline Genre/Audience & Not popular(0) & Popular(1) & Not popular(0) & Popular(1) & \\
\hline All genre & $11,635 \quad(62 \%)$ & $7,122(38 \%)$ & $1,292 \quad(62 \%)$ & $793(38 \%)$ & 20,842 \\
\hline Drama & $4,506 \quad(51 \%)$ & $4,375(49 \%)$ & $502 \quad(50 \%)$ & $485(50 \%)$ & 9,868 \\
\hline Thriller & $2,639 \quad(70 \%)$ & $1,123(30 \%)$ & $295 \quad(70 \%)$ & $124(30 \%)$ & 4,181 \\
\hline Comedy & $3,254 \quad(65 \%)$ & $1,746(35 \%)$ & $(64 \%)$ & $198(36 \%)$ & 5,556 \\
\hline Romance & $1,811 \quad(57 \%)$ & $1,336(43 \%)$ & $196 \quad(56 \%)$ & $154(44 \%)$ & 3,497 \\
\hline Genre/Critics & Not Well-made(0) & Well-made(1) & Not Well-made(0) & Well-made(1) & Total \\
\hline All genre & $5,493 \quad(50 \%)$ & $5,324(50 \%)$ & $590 \quad(49 \%)$ & $612(51 \%)$ & 12,019 \\
\hline Drama & $2,416 \quad(42 \%)$ & $3,306(58 \%)$ & $(42 \%)$ & $363(58 \%)$ & 6,358 \\
\hline Thriller & $1,349 \quad(55 \%)$ & $1,078(45 \%)$ & $(52 \%)$ & $128(48 \%)$ & 2,697 \\
\hline Comedy & $1,898(57 \%)$ & $1,389(43 \%)$ & $(60 \%)$ & $144(40 \%)$ & 3,653 \\
\hline Romance & $1,103 \quad(52 \%)$ & $1,015(48 \%)$ & $107 \quad(45 \%)$ & $129(55 \%)$ & 2,354 \\
\hline Genre/Compound & Not Successful (0) & Successful(1) & Not Successful(0) & Successful(1) & Total \\
\hline All genre & $3,812 \quad(51 \%)$ & $3,586(49 \%)$ & $440 \quad(53 \%)$ & $383(47 \%)$ & 8,221 \\
\hline
\end{tabular}

Table 1: Training and test data set proportion. Class 1 denotes movies with scores greater than 75 . Class 0 denotes movies with scores less than 65 .

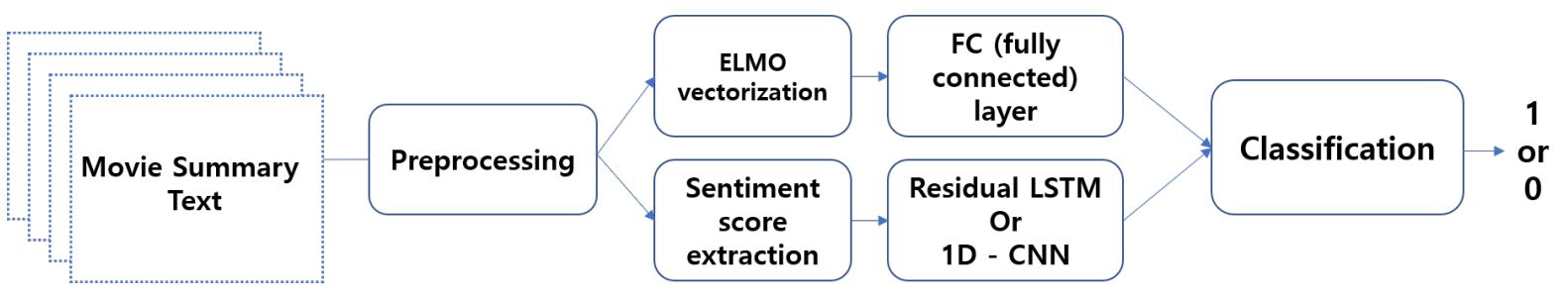

Figure 1: The overall classification procedure

outputs are merged to classify a movie summary into the success or non-success classes.

\subsection{Data}

To evaluate our approach, we used the CMU Movie Summary Corpus (Bamman et al., 2013), which contains crowd-sourced summaries from the real users.

The corpus contains 42,306 movie plot summaries and their metadata such as genre, release date, cast, character traits, etc. However, we use only the plot summary text feature and the genre. The following example summary which consists of 36 sentences and 660 words, shows a part of the plot summary of 'The Avengers' (released in 2012) directed by Joss Whedo.

The Asgardian Loki encounters the Other,the leader of an extraterrestrial race known as the Chitauri. In exchange for retrieving the Tesseract, a powerful energy source of unknown potential,

In the first of two post-credits scenes, the Other confers with his master about the attack on Earth and humanity's resistance; in the second, the Avengers eat in silence at a shawarma restaurant.

We created the classification labels based on the Rotten tomato scores that we crawled from Rotten Tomatoes' website with the Selenium ${ }^{3}$ and Beautiful Soup python packages (Richardson, 2013). These scores serve as a credible indicator of a movie's success (Doshi et al., 2010). We classify movies following the Rotten Tomato rule; if the review score is greater than 75 , the corresponding movie is classified fresh (1); if its score is less than 60 , the movie is classified not fresh $(0)$.

As some movies do not have both the audience and the critics score, we collected 20,842 and 12,019 movie plot summary data for the audience score and for the critic score respectively. The audience score is assessed by ordinary people, we regard the class 1 as representing 'popular' movies and the class 0 as representing 'not popular' movies. Likewise, since the critics score is assessed by professionals in the industry, we consider class 1 as representing 'wellmade' movies and class 0 as representing 'not well-made' movies. Since these scores indicate the popularity and quality of a movie, we define a successful movie as having the combination of

\footnotetext{
${ }^{3}$ https://www.seleniumhq.org/
} 


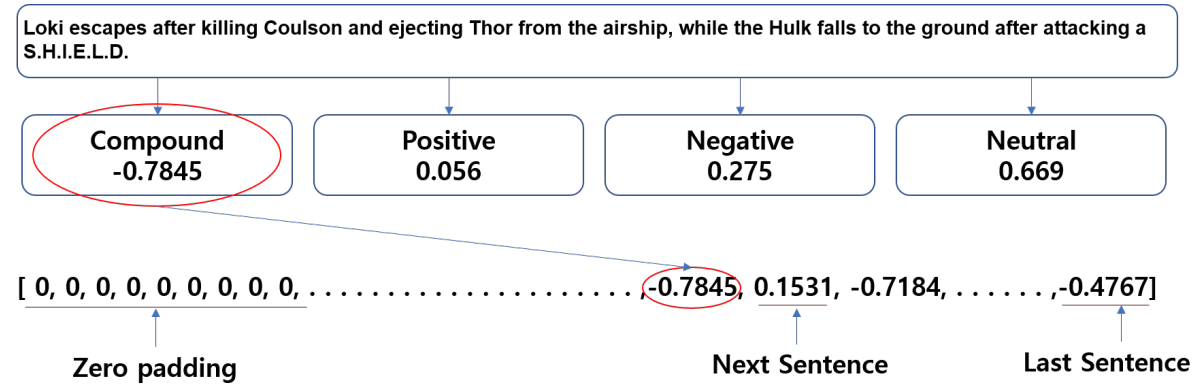

Figure 2: The sentiment vector representation of the movie 'The Avengers'.

these score greater than 75 . Finally, we prepared the third data set considering both of the audience and the critics scores. We define movies with each audience and critics score greater than 75 as 'successful' and less than 60 as 'not successful'.

There are two reasons that the number of instances in the prepared data is less than the number of summaries in the CMU Movie summary corpus. First, movies that have received review scores above 60 and below 75 are filtered out. Second, some movies in the CMU Movie summary corpus have no scores at the Rotten Tomato site.

Table 1 shows the statistics of the data set. The ratio between class 1 and 0 is approximately 6:4 for the audience score and 5:5 for the critics score and the combination of both scores.

The data sets were also divided into different genres, to test whether the genre of a movie has an impact on the prediction of a performance. The table shows the ratios between class 1 and 0 are balanced except for the thriller and comedy genres in the audience score. Since each movie is tagged with multiple genres, the sum of all the number of summaries of each genre is greater than the total number of summaries.

A simple statistical analysis shows that the maximum number of sentences in the longest summary in the train set is 198, the minimum is 1 , and the average is 18.3. The number of words in the largest summary is 4,264 , while that of the shortest summary is 10 . The average is 361.2 words.

\subsection{ELMO embedding}

When the list of sentences representing a movie summary is given as input, the module creates its corresponding word embedding vectors. Traditional word embedding schemes such as Word2vec (Mikolov et al., 2013) and Glove (Pennington et al., 2014) produce a fixed vector for each word. While those embedding methods have been shown effective in many NLP applications, they do not deal with words which mean differently as their contexts vary such as homophones. Thus, We applied a contextualized embedding method that can generate different word vectors depending on the context. ELMO (Peters et al., 2018) is a popular contextualized embedding method, which uses two bidirectional LSTM networks for constructing the vector.

In this work, we utilized the TensorFlow Hub implementation ${ }^{4}$ to represent the word vector. We then fine-tuned the weight for ELMO embedding to gain better performance for the classification task (Perone et al., 2018).

Since the length of the summary varies, we need to set a maximum number of sentences in a summary. We set the maximum number at 198, as it is the number of sentences in the longest summary found in the train set.

\subsection{Sentiment score extraction}

To extract the sentiment score of each sentence, we applied the NLTK's Vader sentiment analyzer (Hutto and Gilbert, 2014) to each sentence. Figure 2 illustrates a part of the sentiment vector representation of the movie 'The Avengers'. A summary is represented as a 198 dimensional vector, where each denotes the sentiment score of a single sentence. A summary shorter than 198 sentences is zero-padded. The highlight of the story (i.e., the conflict and resolution stages) is usually located towards the end of the story. So, we reversed the order as the vector is given as input to the LSTM deep learning model in the next stage which better remember the recent input.

The VADER(Valence Aware Dictionary for sentiment Reasoning) module computes four scores for each sentence: negative, positive, neutral, and compound scores. In this research, we use the

\footnotetext{
${ }^{4}$ https://tfhub.dev/google/elmo/2
} 
compound score ranging from -1 (most negative) to 1 (most positive).

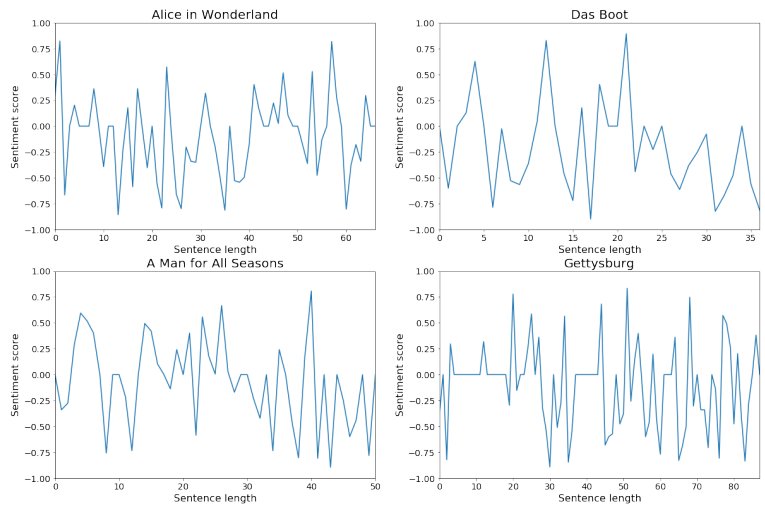

Figure 3: Sentiment flow graphs of successful movies. $\mathrm{X}$ axis denotes the sentence index, and the $\mathrm{Y}$ axis denotes the sentiment score of a sentence normalized between -1 and 1 .
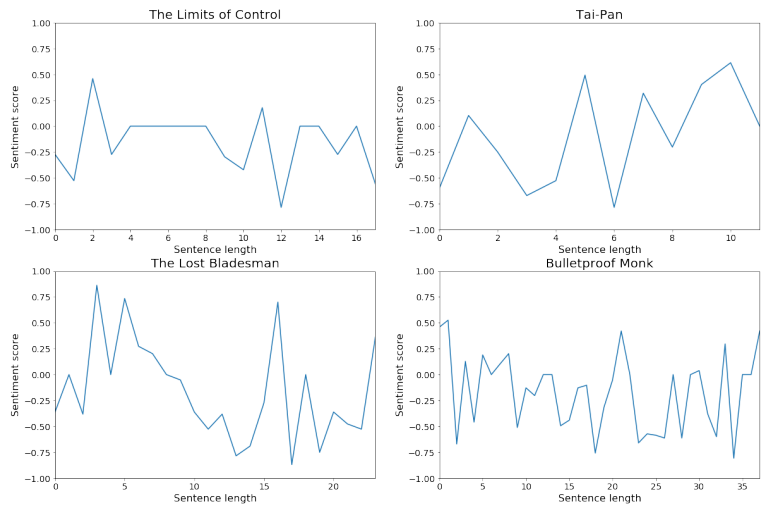

Figure 4: Sentiment flow graphs of unsuccessful movies. $\mathrm{X}$ axis denotes the sentence index, and the $\mathrm{Y}$ axis denotes the sentiment score of a sentence normalized between -1 and 1 .

Figure 3 and Figure 4 depict the sentiment plots of successful movies and unsuccessful movies respectively. The 4 graphs shown in Figure 3 exhibit various patterns of successful movies' sentiment flows. The movie Alice in Wonderland begins and ends positively. On the other hand, the movies Das Boot and A Man for All Seasons begin and end with negatively. The movie Gettysburg shows the reversal of fortune pattern which begins negatively and ends positively. It is commonly noted that these successful movies have frequence sentiment fluctuations. On the other hand, the graphs in Figure 4 illustrate unsuccessful movies' sentiment flows, which exhibit less frequent sentiment fluctuations. Both the movie The Limits of Control and The Lost Bladesman have negative beginning and ending. The movie Tai-Pan begins negatively and ends positively. The movie Bluetproof Monk begins and ends positively, however, its majority sentiment scores are negative while the story is being developed. Therefore, it suggests that the frequency of sentiment changes may signal the success of films. Yet, the polarity of sentiment have a little impact on predicting a movie's success.

\subsection{Classification Models}

We built an ELMO, a merged 1D CNN (Figure 5), and a merged residual LSTM (Figure 6) networks. We establish our baseline by calculating a majority class baseline for comparison.

First, we use deep contextualized word representations created by the ELMO embedding. This network consists of a character embedding layer, a convolutional layer, two highway networks, and two LSTM layers. Each token is converted to a character embedding representation, which is fed to a convolutional layer. Then, it goes through two highway networks to help the deep learning network training. Then, the output is fed to the LSTM layer as input data. The weights of each LSTM hidden layer are combined to generate the ELMO embedding. Finally, a 1024 dimensional ELMO embedding vector is constructed for each sentence, which is put into the 256 dimensional dense network. RELU (Nair and Hinton, 2010) is used as its activation function.

Figure 5 shows the 1D CNN merged network, where the sentiment score vector is given as input to the CNN network. The model consists of two 1D convolutional layers, with 64-size filters and 3 -size kernels. The second CNN layer includes a dropout layer. The next max-pooling layer reduces the learned features to $1 / 4$ of their size. The final flatten layer constructs a single 100-dimensional vector. Then, the output from the ELMO embedding and the output from the CNN model is concatenated and given to the last 1-dense classification layer.

Figure 6 employs two bidirectional LSTM layers which have 128 memory units. The outputs of these layers are added and flattened to create a 50,688 dimensional vector. 50,688 was obtained as the length of the sentences (198) times the size of the vector (256). Then, the next 128 dense layer reduces the vector for the final binary classification. We employed the binary cross-entropy as the loss function and the Adam optimizer. 


\begin{tabular}{|c|c|c|c|c|c|c|c|c|}
\hline Score & Genre & Model & \multicolumn{2}{|c|}{ Recall } & \multicolumn{2}{|c|}{ Precision } & \multicolumn{2}{|c|}{ F1 } \\
\hline & & & 1 & $\mathbf{0}$ & 1 & 0 & 1 & 0 \\
\hline \multirow{15}{*}{ Audience } & \multirow[b]{3}{*}{ All } & ELMO & 0.54 & 0.81 & 0.64 & 0.74 & 0.58 & 0.78 \\
\hline & & CNN & 0.38 & 0.90 & 0.70 & 0.70 & 0.49 & 0.79 \\
\hline & & LSTM & 0.56 & 0.67 & 0.51 & 0.71 & 0.53 & 0.69 \\
\hline & \multirow{3}{*}{ Drama } & ELMO & 0.62 & 0.73 & 0.69 & 0.67 & 0.66 & 0.70 \\
\hline & & LSTM & 0.79 & 0.39 & 0.56 & 0.66 & 0.65 & 0.49 \\
\hline & & CNN & 0.77 & 0.48 & 0.59 & 0.68 & 0.67 & 0.56 \\
\hline & \multirow[b]{3}{*}{ Thriller } & ELMO & 0.39 & 0.91 & 0.65 & 0.78 & 0.48 & 0.84 \\
\hline & & $\mathrm{CNN}$ & 0.41 & 0.79 & 0.45 & 0.76 & 0.43 & 0.77 \\
\hline & & LSTM & 0.60 & 0.70 & 0.45 & 0.80 & 0.52 & 0.75 \\
\hline & \multirow[b]{3}{*}{ Comedy } & ELMO & 0.31 & 0.94 & 0.73 & 0.71 & 0.43 & 0.81 \\
\hline & & CNN & 0.41 & 0.83 & 0.57 & 0.72 & 0.48 & 0.77 \\
\hline & & LSTM & 0.62 & 0.63 & 0.48 & 0.75 & 0.54 & 0.68 \\
\hline & \multirow[b]{3}{*}{ Romance } & ELMO & 0.63 & 0.68 & 0.61 & 0.70 & 0.62 & 0.69 \\
\hline & & $\mathrm{CNN}$ & 0.57 & 0.67 & 0.58 & 0.67 & 0.58 & 0.67 \\
\hline & & LSTM & 0.55 & 0.71 & 0.60 & 0.67 & 0.57 & 0.69 \\
\hline
\end{tabular}

Table 2: The evaluation results for the audience score. The best performances in F1 score are in bold.

\begin{tabular}{|c|c|c|c|c|c|c|c|c|}
\hline Score & Genre & Model & \multicolumn{2}{|c|}{ Recall } & \multicolumn{2}{|c|}{ Precision } & \multicolumn{2}{|c|}{ F1 } \\
\hline & & & 1 & $\mathbf{0}$ & 1 & O & 1 & $\mathbf{0}$ \\
\hline \multirow{15}{*}{ Critics } & \multirow{3}{*}{ All } & ELMO & 0.72 & 0.60 & 0.65 & 0.68 & 0.69 & 0.63 \\
\hline & & CNN & 0.76 & 0.56 & 0.64 & 0.69 & 0.70 & 0.62 \\
\hline & & LSTM & 0.71 & 0.63 & 0.66 & 0.68 & 0.69 & 0.65 \\
\hline & \multirow{3}{*}{ Drama } & ELMO & 0.79 & 0.47 & 0.66 & 0.63 & 0.72 & 0.53 \\
\hline & & CNN & 0.79 & 0.46 & 0.66 & 0.62 & 0.72 & 0.53 \\
\hline & & LSTM & 0.71 & 0.50 & 0.65 & 0.57 & 0.68 & $\mathbf{0 . 5 3}$ \\
\hline & \multirow[b]{3}{*}{ Thriller } & ELMO & 0.65 & 0.72 & 0.67 & 0.69 & 0.66 & 0.71 \\
\hline & & CNN & 0.68 & 0.77 & 0.73 & 0.73 & 0.70 & 0.75 \\
\hline & & LSTM & 0.64 & 0.76 & 0.71 & 0.70 & 0.67 & 0.73 \\
\hline & \multirow[b]{3}{*}{ Comedy } & ELMO & 0.61 & 0.77 & 0.63 & 0.75 & 0.62 & 0.76 \\
\hline & & CNN & 0.52 & 0.82 & 0.65 & 0.73 & 0.58 & 0.77 \\
\hline & & LSTM & 0.49 & 0.80 & 0.62 & 0.71 & 0.55 & 0.75 \\
\hline & \multirow{3}{*}{ Romance } & ELMO & 0.64 & 0.62 & 0.67 & 0.58 & 0.65 & 0.60 \\
\hline & & CNN & 0.64 & 0.61 & 0.66 & 0.59 & 0.65 & 0.60 \\
\hline & & LSTM & 0.71 & 0.50 & 0.63 & 0.59 & 0.67 & 0.54 \\
\hline
\end{tabular}

Table 3: The evaluation results for the critics score. The best performances in F1 score are in bold.

\begin{tabular}{|c|c|c|c|c|c|c|c|c|}
\hline \multicolumn{2}{|c|}{ Score } & Genre & Model & \multicolumn{2}{c|}{ Recall } & \multicolumn{2}{c|}{ Precision } & \multicolumn{2}{|c|}{ F1 } \\
\hline \multicolumn{3}{|c|}{} & $\mathbf{1}$ & $\mathbf{0}$ & $\mathbf{1}$ & $\mathbf{0}$ & $\mathbf{1}$ & $\mathbf{0}$ \\
\hline \multirow{3}{*}{ Audience\&Critics } & \multirow{3}{*}{ All genre } & ELMO & 0.67 & 0.74 & 0.69 & 0.72 & $\mathbf{0 . 6 8}$ & $\mathbf{0 . 7 3}$ \\
\cline { 3 - 10 } & & CNN & 0.68 & 0.70 & 0.64 & 0.67 & 0.66 & 0.69 \\
\cline { 3 - 9 } & LSTM & 0.68 & 0.67 & 0.64 & 0.71 & 0.66 & 0.69 \\
\hline
\end{tabular}

Table 4: The evaluation results for the audience $\&$ critics score. The best performances in F1 score are in bold. 


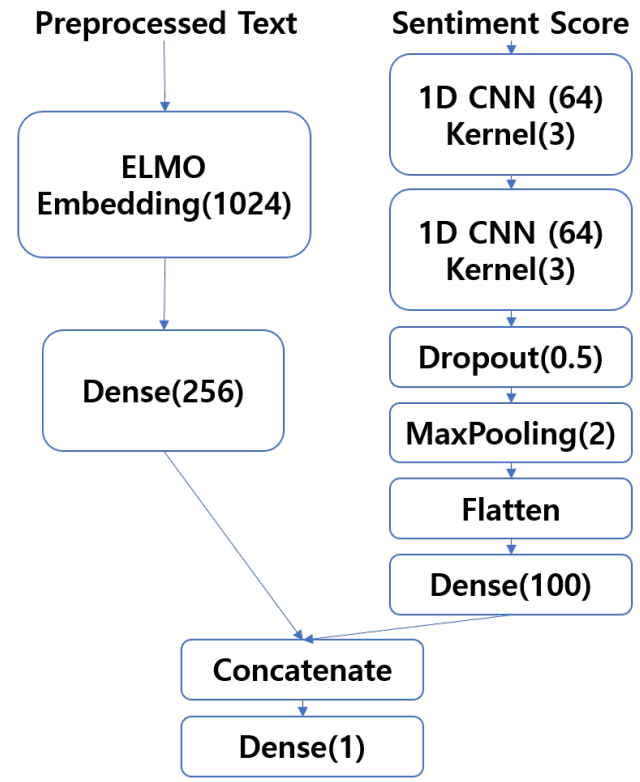

Figure 5: A merged 1D CNN

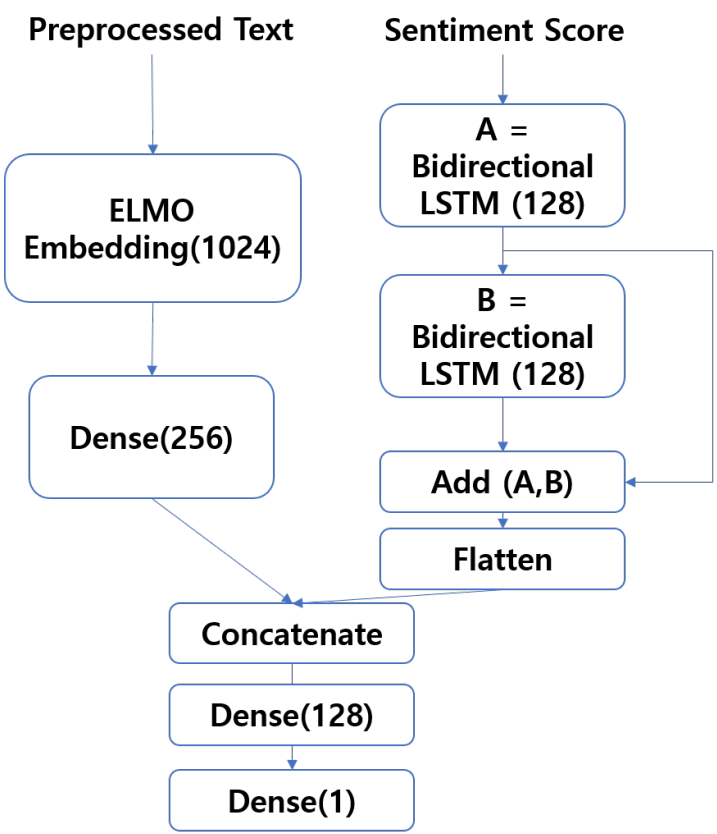

Figure 6: A merged bidirectional residual LSTM

\section{Evaluation Results}

We evaluated the classification performance of our approach for the audience score and for the critics score. We also inspected the performance based on the movie genre. We report the performance in terms of recall, precision, and $\mathrm{F} 1$ scores.

\subsection{The Results}

Table 2 shows the performance result for the audience score. We use the F1 score as the primary metric for comparison as it is the harmonic means of recall and precision. Overall, the classification performance of 'not popular' movies better than that of 'popular' ones. The CNN model performed best in 'all genre' with F1 of 0.79 , which is 0.17 higher than the majority class baseline (F1 of 0.62). The ELMO model outperformed best in the genres of drama, thriller, comedy, and romance. On the contrary, the ELMO model had the highest performance for 'popular' at 0.58 and 0.62 in overall and romance genre respectively, while LSTM and CNN had the highest performance in the rest of the genre

Table 3 summarizes the evaluation results for the critics score.

For all the genres, the deep learning models outperform the majority class baseline (F1 score $=0.51$ ) for predicting 'well-made' movies producing its highest $\mathrm{F} 1$ of 0.70 . The $\mathrm{CNN}$ model achieved the highest F1 score of 0.72 in predicting 'well-made' drama movies when its majority class baseline performance is 0.58 . In the thriller, the CNN model also outperformed the baseline (F1 score $=0.52$ ) producing an F1 score of 0.75 . The LSTM model achieved the best performance in predicting 'not well-made' movies, and yet the score is low- 0.65 .

Inspection of the genre-specific F1 score shows that the best performance was obtained from CNN model when predicting 'not well-made' movies for the comedy genre (F1 score of 0.77).

Finally, Table 4 shows the results when our approach is applied to the combined score. The ELMO embedding model outperforms the majority class baseline and the other models, achieving $\mathrm{F} 1$ scores of 0.68 and 0.73 when predicting 'successful' and 'not successful' movies respectively.

\subsection{Discussions}

Overall, the results suggest that the merged deep learning models proposed in this paper outperform the majority class baseline.

For the audience score, the performance results of predicting 'not popular' movies outperform that of predicting 'popular' movies. This may suggest that using the textual summary only is limited in predicting 'popular' movies. When inspecting the results genre-wise, the precision of predicting 'not popular' movies for the thriller and the comedy genres yields the best performance when the LSTM model is used along with the sentiment score. On the other hand, the ELMO model out- 
performs the merged deep learning models that employ the sentiment score in predicting 'popular' movies with significant difference.

The CNN model produces a F1 score higher than ELMo does in the thrillers and comedy genres and in the drama genre for 'popular' movies.

In case of the critics score, the overall performance was inferior to that of the audience score. Inspection of the F1 score of each genre shows that predicting 'not well-made' movies in the thriller and the comedy genre achieved the best performance ( 0.75 and 0.77 respectively) when the $\mathrm{CNN}$ model was used along with the sentiment score. Generally, the CNN or LSTM models have shown F1 scores higher than the ELMO models at predicting well-made movies using the critics score except the drama genre.

Then, employing the ELMO model outperforms other models that used the sentiment score as well. This may suggest that words are the primary determiner of predicting a movie' success.

The research work by Eliashberg et al. Eliashberg et al. (2007) is most similar to our work. Their evaluation achieved the F1 score of 0.5 (recomputed from the evaluation metrics reported) in predicting a movie's success using the CART (Bootstrap Aggregated Classification and Regression Tree) model and the movie spoiler text which is 4-20 pages long. Although our result appear to be superior to their work in terms of yielding higher F1 score, it is not directly comparable since the data sets and the evaluation metrics are different.

\section{Related work}

The prediction of movie box office results has been actively researched (Rhee and Zulkernine, 2016; Eliashberg et al., 2007, 2010, 2014; Sharda and Delen, 2006; Zhang et al., 2009; Du et al., 2014).

Most researches predict a movie's success using various factors such as SNS data, cost, critics ratings, genre, distributor, release season, and the main actors award history, etc (Mestyán et al., 2013; Rhee and Zulkernine, 2016; Jaiswal and Sharma, 2017). This means that the prediction is made in the later stages of movie production, when the movie has already been produced and released.

The evaluation carried out in (Jaiswal and Sharma, 2017) achieved the highest performance with F1 score of 0.79 , which is recomputed from the evaluation metrics reported. However, this performance is not directly comparable to our result, since their work employed a small data set which consists of 557 movies and was based on a different genre (i.e., Bollywood movie). Their work employs rich feature such as YouTube statistics, lead actor, actress and director ratings, critics reviews, which are mostly available only after the movie is produced. Therefore, movie distributors and investors cannot rely on this approach when they need to make an investment decision.

To overcome this problem, our approach relies on only the plot summary, which can assist the investors in making their invest decisions in the very early stages when they only have the written movie script.

\section{Conclusions}

In this paper, we propose a deep learning based approach utilizing the ELMO embedding and sentiment scores of sentences for predicting the success of a movie, based only on a textual summary of the movie plot. To test the efficacy of our approach, we prepared our evaluation data sets: movie plot summaries gathered from the CMU Movie Summary Corpus and their review scores from a movie review website.

Since these plot summaries were obtained from Wikipedia, where the data are crowd sourced voluntarily. Hence, some movie summaries may have been written by people who like or value the movie. This may complicate our task to predict the movie's success only from the summary. We built three deep learning models: an ELMO embedding and two merged deep learning models (a merged 1D CNN network and a merged residual bidirectional LSTM network).

The evaluation results show that our deep learning models outperform the majority class baseline.

For the combination of the audience and the critics scores, the majority class baseline is F1 of 0.53 for 'not successful', and 0 for 'successful'. Our best model obtained the highest F1 score of 0.68 for predicting 'successful' movies and that of 0.70 for predicting 'not successful' movies were obtained.

Considering that only textual summaries of the movie plot are used for the predictions, the study results are promising. Forecasting the popularity and success of movies only with their textual descriptions of the plot, will aid the decision-making 
in funding movie productions.

It seems that predicting 'not popular' or 'not successful' movies performs better than that of predicting 'popular' or 'successful' movies. Predicting unsuccessful movies can be useful for the Internet Protocol television (IPTV) content providers such as Netflix. Whereas tens of thousands of TV contents are made available, only a small portion of them are actually consumed ( $\mathrm{Re}$ format and Yager, 2014). Therefore, our approach can be used to filter out such contents that are not appealing to the content viewers.

For future work, we will further investigate the efficacy of our approach in the thriller and the comedy genes, which presented the best performances. In addition, we will extend our model to deal with the magnitude of a movie's success. For this, linear regression models can be applied to predict different levels of success.

\section{Acknowledgement}

This work was supported by the National Research Foundation of Korea(NRF) grant funded by the Korea government(MEST) (No. 2019R1A2C1006316). This research was supported by Basic Science Research Program through the National Research Foundation of Korea(NRF) funded by the Ministry of Education(No. 2016R1D1A1B03933002).

\section{References}

David Bamman, Brendan OConnor, and Noah A Smith. 2013. Learning latent personas of film characters. In Proceedings of the 51 st Annual Meeting of the Association for Computational Linguistics (Volume 1: Long Papers), volume 1, pages 352-361.

Arthur De Vany and W David Walls. 1999. Uncertainty in the movie industry: Does star power reduce the terror of the box office? Journal of cultural economics, 23(4):285-318.

Lyric Doshi, Jonas Krauss, Stefan Nann, and Peter Gloor. 2010. Predicting movie prices through dynamic social network analysis. Procedia-Social and Behavioral Sciences, 2(4):6423-6433.

Jingfei Du, Hua Xu, and Xiaoqiu Huang. 2014. Box office prediction based on microblog. Expert Systems with Applications, 41(4):1680-1689.

Jehoshua Eliashberg, Sam K Hui, and Z John Zhang. 2007. From story line to box office: A new approach for green-lighting movie scripts. Management Science, 53(6):881-893.
Jehoshua Eliashberg, Sam K Hui, and Z John Zhang. 2014. Assessing box office performance using movie scripts: A kernel-based approach. IEEE Transactions on Knowledge and Data Engineering, 26(11):2639-2648.

Jehoshua Eliashberg, SK Hui, and SJ Zhang. 2010. Green-lighting Movie Scripts: Revenue Forecasting and Risk Management. Ph.D. thesis, Ph. D. thesis, University of Pennsylvania.

Clayton J Hutto and Eric Gilbert. 2014. Vader: A parsimonious rule-based model for sentiment analysis of social media text. In Eighth international AAAI conference on weblogs and social media.

Sameer Ranjan Jaiswal and Divyansh Sharma. 2017. Predicting success of bollywood movies using machine learning techniques. In Proceedings of the 10th Annual ACM India Compute Conference on ZZZ, pages 121-124. ACM.

Márton Mestyán, Taha Yasseri, and János Kertész. 2013. Early prediction of movie box office success based on wikipedia activity big data. PloS one, 8(8):e71226.

Tomas Mikolov, Kai Chen, Greg Corrado, and Jeffrey Dean. 2013. Efficient estimation of word representations in vector space. arXiv preprint arXiv:1301.3781.

Vinod Nair and Geoffrey E Hinton. 2010. Rectified linear units improve restricted boltzmann machines. In Proceedings of the 27th international conference on machine learning (ICML-10), pages 807-814.

Jeffrey Pennington, Richard Socher, and Christopher Manning. 2014. Glove: Global vectors for word representation. In Proceedings of the 2014 conference on empirical methods in natural language processing (EMNLP), pages 1532-1543.

Christian S Perone, Roberto Silveira, and Thomas S Paula. 2018. Evaluation of sentence embeddings in downstream and linguistic probing tasks. arXiv preprint arXiv:1806.06259.

Matthew E Peters, Mark Neumann, Mohit Iyyer, Matt Gardner, Christopher Clark, Kenton Lee, and Luke Zettlemoyer. 2018. Deep contextualized word representations. arXiv preprint arXiv:1802.05365.

Marek Z Reformat and Ronald R Yager. 2014. Suggesting recommendations using pythagorean fuzzy sets illustrated using netflix movie data. In International Conference on Information Processing and Management of Uncertainty in Knowledge-Based Systems, pages 546-556. Springer.

Travis Ginmu Rhee and Farhana Zulkernine. 2016. Predicting movie box office profitability: a neural network approach. In 2016 15th IEEE International Conference on Machine Learning and Applications (ICMLA), pages 665-670. IEEE. 
Leonard Richardson. 2013. Beautiful soup. Crummy: The Site.

Shaiwal Sachdev, Abhishek Agrawal, Shubham Bhendarkar, Bakshi Rohit Prasad, and Sonali Agarwal. 2018. Movie box-office gross revenue estimation. In Recent Findings in Intelligent Computing Techniques, pages 9-17. Springer.

Ramesh Sharda and Dursun Delen. 2006. Predicting box-office success of motion pictures with neural networks. Expert Systems with Applications, 30(2):243-254.

Li Zhang, Jianhua Luo, and Suying Yang. 2009. Forecasting box office revenue of movies with bp neural network. Expert Systems with Applications, 36(3):6580-6587. 